
\title{
$\begin{array}{ll}\text { Research Square } & \begin{array}{l}\text { Preprints are preliminary reports that have not undergone peer review. } \\ \text { They should not be considered conclusive, used to inform clinical practice, } \\ \text { or referenced by the media as validated information. }\end{array}\end{array}$
}

\section{Study on the Mechanism of Buyang Huanwu Decoction for the treatment of Ischemic Stroke Based on Network Pharmacology}

Kai Wang ( $\nabla$ wang65k86@163.com)

College of Pharmacy, Shaanxi University of Chinese Medicine

Lu Lei

Department of Pharmacy, Xijing Hospital, Fourth Military Medical University

Jinyi Cao

Department of Pharmacy, Xijing Hospital, Fourth Military Medical University

Yi Qiao

Department of Pharmacy, Xijing Hospital, Fourth Military Medical University

\section{Ruimin Liang}

College of Pharmacy, Shaanxi University of Chinese Medicine

Jialin Duan

Department of Pharmacy, Xijing Hospital, Fourth Military Medical University

Zhijun Feng

Department of Pharmacy, Xijing Hospital, Fourth Military Medical University

Yi Ding

Department of Pharmacy, Xijing Hospital, Fourth Military Medical University

Yang Ma

Department of Pharmacy, Xijing Hospital, Fourth Military Medical University

Enhu Zhang

College of Pharmacy, Shaanxi University of Chinese Medicine

Zhifu Yang

Department of Pharmacy, Xijing Hospital, Fourth Military Medical University

\section{Research}

Keywords: Traditional Chinese medicine, Buyang Huanwu Decoction, Network Pharmacology, Cerebral ischemia, Pharmacological mechanism

Posted Date: April 28th, 2020

DOI: https://doi.org/10.21203/rs.3.rs-24632/v1

License: () (1) This work is licensed under a Creative Commons Attribution 4.0 International License. Read Full License 


\section{Abstract}

Background: Buyang Huanwu Decoction (BYHWD) is one of the representative prescriptions for tonifying qi and promoting blood circulation. This formula has been widely used in Chinese clinical practice for treatment and prevention of ischemic cerebral vascular diseases. However, the mechanism and active compounds of BYHWD used in clinical practice for ischemic stroke are not well understood. The purpose of this study was to understand the potential active components of BYHWD and further explore its mechanism of improving ischemic stroke.

Methods: This study was based on network pharmacology and bioinformatics analysis. The compounds of BYHWD were obtained from public databases. Oral bioavailability as well as drug-likeness were screened by using absorption, distribution, metabolism, and excretion (ADME) criteria. Then, components of BYHWD, candidate targets of each component and known therapeutic targets of ischemic cerebral were collected. A network of compound-target genes and compound-ischemic cerebral was established by means of network pharmacology data sources. The enrichment of key targets and pathways was analyzed by using string database and DAVID database. In addition, we verified three key targets predicted by western blot analysis (IL6, VEGFA and HIF1A).

Results: Network pharmacology analysis results of BYHWD identified 7 herbs, 42 compounds and 79 target genes associated to cerebral ischemia. The 10 key compounds were baicalein, beta-carotene, Baicalin, kaempferol, luteolin, quercetin, hydroxysafflor yellow A, isorhamnetin, Bifendate, formononetin,Calycosin, AstragalosidelV, Stigmasterol, sitosterol, Z-ligustilide, Dihydrocapsaicin. Core genes in this network were IL6, TNF, VEGFA, HIF1A, MAPK1, MAPK3, JUN, STAT3, IL1B and IL10. And pathways TNF, IL-17, Apoptosis, PI3K-Akt, Toll-like receptor, MAPK, NF-kappa B and HIF-1 signaling pathway, etc. related to ischemic stroke were identified. In vitro experiments, The results showed that compared with the control group (no treatment), BYHWD could significantly inhibit the expression of IL6 and increased the expression of HIF1A and VEGFA.

Conclusions: Network pharmacology analysis can reveal close interactions between multi-components and multi-targets, and enhance our understanding of the potential effects of BYHWD in cerebral ischemia.

\section{Background}

Ischemic stroke is an acute cerebrovascular disease, also known as cerebral ischemia. It has the characteristics of high disability and high mortality[1, 2]. According to statistics compiled by the American heart association (AHA) in collaboration with the national institutes of health and other government agencies, stroke has become the third most common cause of death worldwide after cancer and heart disease in recent years, placing a huge psychological and financial burden on many families[3].

Buyang Huanwu Decoction (BYHWD) is one of the representative prescriptions for tonifying qi and promoting blood circulation in the "Correction on Errors in Medical Classic" by Wang Qingren, a famous doctor in the Qing Dynasty. It is composed of: Radix Astragali, the dried roots of Astragalus membranaceus (Fisch.) Bge; Angelicae sinensis Radix, the dried roots of Angelica sinensis(Oliv.)Diels; Paeoniae Radix Rubra, the dried roots of Paeonia lactiflora Pall; Chuangxiong Rhizoma, the dried rhizomes of Ligusticum chuanxiong Hort; Persicae Semen, the dried seeds of Prunus persicalL."Batsch; Carthami Flos, the

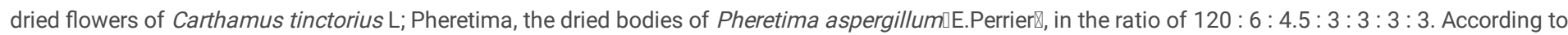
the traditional Chinese medicine literature, BYHWD was used to promote blood circulation, and activate energy (qi) flow through energy meridians. This formula has been widely used in Chinese clinical practice for treatment and prevention of ischemic cardio-cerebral vascular diseases[4]. Many researches show that BYHWD has notable curative effectiveness in ischemic stroke and other vascular diseases[5]. However, the exact mechanism of BYHWD in improving ischemic stroke and its active components remain unclear. As such, there is a great interest in identifying the active compounds and molecular targets in BYHWD acting on ischemic stroke. The present study aimed to identify the effective constituents of BYHWD and to further explore its action mechanisms in the amelioration of ischaemic stroke.

Traditional Chinese medicine compound has the characteristics of "multi-component, multi-channel, multi-target". It is a product of traditional Chinese medicine under the guidance of holistic view and dialectical theory. It has unique advantages for thetreatment of complex diseases, but because of the complex composition of traditional Chinese medicine compound to make its modernization process slow. Network pharmacology, a novel method which combined the system network analysis and the pharmacology, could clarify the synergistic effects and underlying mechanisms among the networks of compound-compound, compound-target and target-disease in the molecular level, which let us know the interactions among the compounds, genes, proteins and diseases. Due to its integrity and systematicness, which are consistent with the overall view of TCM and the principle of dialectical theory, it has been widely applied in TCM research[6]. Therefore, this study applied network pharmacology method to study and discuss the mechanism of BYHWD in the treatment of ischemic stroke, providing reference and theoretical basis for its experimental research and clinical application.

In this study, information on compounds in herbal medicines of BYHWD was acquired coming from public databases, and oral bioavailability (OB) and druglikeness (DL) were evaluated utilizing ADME standards. Then, components of BYHWD, candidate targets of each component and known therapeutic targets of ischemic stroke were collected. By utilizing these data, compounds of BYHWD-compound targets and compounds - ischemic stroke target networks were established via network pharmacology databases. Key targets and pathway enrichment were analyzed by STRING database and DAVID database. In addition, we verified three of the key targets predicted in the network by western blot analysis. The workflow of this study on BYHWD against ischemic stroke based on network pharmacology was drawn (Fig. 1).

\section{Materials And Methods}

\subsection{Chemical databases collection of BYHWD}


The compound of buyang huanwu decoction was collected from two phytochemical databases: Traditional Chinese Medicine Systems Pharmacology Database (TCMSP, http://ibts.hkbu.edu.hk/LSP/tcmsp.php) and TCM Database@Taiwan (http://tcm.cmu.edu.tw/). Bidimensional chemical structures were also gotten from NCBI PubChem (http://pubchem.ncbi.nlm.nih.gov/).

\subsection{Pharmacokinetic ADME evaluation}

OB represents the ability of a compound to circulate in the body after oral administration. OB can show whether the active compounds in a formula can be delivered through out the body and produce a physicochemical effect. DL is an indicator for determining the similarity or likeness of a compound, and its physicochemical properties, with conventional drugs. DL can help determine if a certain compound has a therapeutic effect[7]. All compounds were selected using the in silico integrative ADME model administered by the TCMSP Database. Chemicals without ADME information were removed from the final list. The $A D M E$ system used in this study includes predict $O B$ and $D L$, and compounds were retained only if $O B \geq 30$ and $D L \geq 0.18$ to satisfy criteria suggested by the TCMSP database[8].

\subsection{Target genes related to the identified compounds}

Collected all the genes related to the compound from the database, The official names of gene were taken from UniProt (http://www.uniprot.org/) by confining the species to'Homo sapiens', Subsequently, various ID forms of the targets were transformed into UniProt IDs.

\subsection{Target genes collection of cerebral ischemia}

Information on cerebral ischemia associated target genes was collected from the therapeutic targets database (TTD; http://bidd.Nus.edu.sg/group/cjttd/TTD_HOME.asp) and DisGeNETv6.0(http://www.disgenet.org/web/DisGeNET/menu/home),and simply “Homo sapiens"proteins connected to cerebral ischemia were selected.

\subsection{Construction of networks and pathway analyses}

The constructed herbal-chemical-protein networks were visualized using Cytoscape ver.3.6.1 (http://www.cytoscape.org/), Nodes in networks represent herbs and chemicals, and edges indicate interactions between herbs and chemicals, and between chemicals and target genes[9]. The functional pathways of BYHWD related to cerebral ischemia diseases were analyzed using Kyoto Encyclopedia of Genes and Genomes (KEGG) pathway (http://www.genome.jp/kegg/pathway.html) and gene ontology (GO) enrichment evaluation based upon the database for annotation, Visualization and Integrated Discovery(DAVID) version 6.8 (https://david.ncifcrf.gov/). The value of $\mathrm{P}<0.05$ was screened from the enrichment analysis results.

\subsection{Protein-Protein interaction (PPI) analyses}

The screened drug component-disease common target was imported into the STRING (https://string-db.org/) database to build the protein-protein interaction (PPI) network model,and the protein category was set as "Homo sapiens", the minimum interaction threshold was set as the medium "highest confidence" (> 0.9 ), and the PPI network was obtained with the default setting of other parameters. In the network, thesize of the nodes represents the size of the degree.The higher the degree, the better the correlation between protein and therapeutic mechanism[10].

\subsection{Pharmacological experimental verification}

\subsubsection{Materials}

Buyang Huanwu Decoction is a granule made up of Astragali Radix, Angelicae sinensis Radix, Paeoniae Radix Rubra, Chuangxiong Rhizoma, Persicae Semen, Carthami Flos and Pheretima according to the ratio of $120: 6: 4.5: 3: 3: 3: 3$ (Manufacturer: Guangdong Yifang pharmaceutical co. LTD. Product batch:7070352). The rat Brain Microvascular Endothelial cells (BMECs) were purchased from the Cell Biologics Company (\#C57-6023, Chicago, IL). Primary antibody against $\beta$-actin, VEGFA, HIF1A, IL6 and Secondary antibody were all purchased from Abcam technology (Cambridge, UK). All other reagents are analytical reagents and commercially available.

\subsubsection{Cell culture and treatments}

Cells were divided into three groups: control group, OGD group and OGD group treated with $\mathrm{HH}(100 \mu \mathrm{g} / \mathrm{mL})$. Oxygen Glucose Deprivation (OGD) was established as follow: cells were rinsed once with glucose-free DMEM (Gibco, Rockville, MD) and transferred to an anaerobic chamber (Forma Scientific, Waltham, MA) containing a gas mixture composed of $7 \% \mathrm{CO} 2$ and $93 \% \mathrm{~N} 2 \mathrm{for} 6 \mathrm{~h}$ at $37{ }^{\circ} \mathrm{C}$. Then, the cells were returned to the normal culture condition . Control BMECs were cultured in complete DMEM under normal conditions. BYHWD groups were treated with $80 \mu \mathrm{g} / \mathrm{mL}$ BYHWD (dissolved in complete DMEM and filtered with a $0.22 \mu \mathrm{m}$ membrane filter) for $12 \mathrm{~h}$. The control group was treated without BYHWD.

\subsubsection{Western blot assay}

BMECs were lysed with cold RIPA buffer (Rockford, IL, USA) for 30 min. The whole cell lysates were fractionated by $10 \%$ sodium dodecyl sulfate polyacrylamide gel electrophoresis (SDS-PAGE) and then transferred onto PVDF membranes (Millipore, USA). The membranes were blocked with $5 \%$ bovine serum albumin (BSA) and incubated overnight with primary antibodies against VEGFA (1:1000,rabbit), HIF1A (1:1000,rabbit), IL6 (1:1000,rabbit) at $4^{\circ} \mathrm{C}$. Subsequently, membranes were incubated with secondary antibody at a 1:10000 dilution at $37^{\circ} \mathrm{C}$ for $1 \mathrm{~h}$. The blots were visualized with ECL-Plus reagent (Santa Cruz, USA) and analyzed with Quantity One System image analysis software (Bio-Rad, USA).

\subsubsection{Statistical analysis}

The results were expressed as mean \pm standard deviation (S.D.). Differences between multiple groups were analyzed by One-way analysis of variance (ANOVA) and differences between two groups were analyzed using the t-test. Significant difference was considered when $\mathrm{P}<0.05$. 


\section{Results}

\subsection{Compounds in herbal medicines}

BYHWD consists of seven herbal medicines, namely Astragali Radix, Angelicae sinensis Radix, Paeoniae Radix Rubra, Chuangxiong Rhizoma, Persicae Semen, Carthami Flos and Pheretima. A total of 102 compounds were identified in BYHWD, among which 21 were Astragali Radix, 23 were Carthami Flos, 4 were Angelicae sinensis Radix, 29 were Paeoniae Radix Rubra, 9 were Chuangxiong Rhizoma, 23 were Persicae Semen and 5 were Pheretima. All identified compounds were subjected to ADME screening, among compounds below these requirements, six (Hydroxysafflor yellow A, AstragalosidelV, Ferulic acid, Ligustrazine, Z-ligustilide, Linoleic acid) were considered bioactive compounds. These compounds are the major components of BYHWD, and their effects on cerebral ischemia is have been investigated previously[11-16]. Remove duplicate components and remove components with ambiguous targets. The composition table of BYHWD was obtained, as shown in Table 1.

\subsection{Combination of compound target genes and cerebral ischemia targetgenes}

A total of 518 genes and compounds related to BYHWD were obtained from the database. A total of 274 genes related to cerebral ischemia were retrieved from the TTD and DisGeNETv6.0 database, and 79 overlapping genes were pinpointed through matching the mentioned 518 compounds genes with diseaseassociated genes (Fig. 2).

\subsection{Potential target genes and network analysis}

The constructed herbal-chemical-protein networks were visualized using Cytoscape, this network of 7 herbs 42 compounds and 79 target genes has 129 nodes and 490 edges (Fig. 3). Particularly, among the 16 compounds (baicalein, beta-carotene, Baicalin, kaempferol, luteolin, quercetin, hydroxysafflor yellow A, isorhamnetin, Bifendate, formononetin, Calycosin, AstragalosidelV, Stigmasterol, sitosterol, Z-ligustilide, Dihydrocapsaicin) were linked to more than five genes. Additionally, 33 genes (PTGS2, MMP9, NOS2, MAPK1, MAPK3, TNF, EGFR, APP, JUN, TP53, IL6, IL2, STAT3, NOS3, IL10, CYCS, VEGFA, HIFA, BCL2, CASP9, CAT, PARP1, CASP3, CCL2, MPO, NFE2L2, CSF2, RELA, ALB, NFKBIA, PPARG, NR3C2, ICAM1) were regulated by more than four compounds. The compound-target gene network showed intimate communications between several components and multiple targets, and facilitated a better understanding of the possible curative results of cerebral ischemia.

\subsection{PPI network analysis of target genes}

The drug component-disease common target was imported into the STRING database to construct the PPI network model of protein-protein interaction. With a interaction score of $>0.9$ was selected, the network of PPI relationships has 79 nodes and 327 edges, the average node degree was 8.28 , and PPI enrichment p-value was < 1.0e-16 (Fig. 4A). Cytoscape3.6.1 was used to analyze the degree and combined score of PPI network topological eigenvalues, the degree of node color and size reaction center, the edge thickness and colordepth reflect the combined score. The highest twenty target genes with a high degree of connectivity were selected as the hub genes for cerebral ischemia (Fig. 4B).

\subsection{GO and KEGG enrichment analysis of potential target genes}

To explore the molecular mechanism of BYHWD on cerebral ischemia, GO enrichment analysis and KEGG pathway enrichment of the 79 potential targets were executed. GO enrichment analysis results included biological process (BP), molecular function (MF) and cellular component (CC). Analyze in the DAVID database, take a value of $\mathrm{P}<0.05$, the results showed that the main biological processes have inflammatory response, negative regulation of apoptosis, angiogenesis, positive regulation of NF-kB transcription factor activity, apoptosis process, immuneresponse, and response to oxidative stress. The molecular function is mainly related to DNA binding, transcription factor activity, heparin binding, heme binding, cytokine activity and growth factor activity. Cell component analysis showed that nucleus and extracellular spacefor the higher proportion. An introduction of the GO analysis was discovered with the top 6 enriched conditions in the BP, CC and MF categories (Fig. 5) and the remarkable 20 biological processes in which they are involved were presented in Table 2. Subsequently, to examine the signaling pathways and functions of these target genes, we performed functional enrichment analysis using KEGG pathways. Obtained the signal pathway with statistical significance $(\mathrm{p}<0.05)$, and target genes were found to interact mainly with the TNF signaling pathway, IL-17 signaling pathway, PI3K-Akt signaling pathway, Toll-like receptor signaling pathway, MAPK signaling pathway, NF-kB signaling pathway, and HIF-1 signaling pathway. Therefore, these signaling pathways appear to be closely associated with the potential effects of BYHWD against cerebral ischemia. Identified target genes were listed in Table 3 and take the top 20 signal pathways and draw KEGG bubble diagram (Fig. 6A). Additionally, main functional annotation clustersranked by the Biocarta functional annotation cluster tool are presented in Fig. 6B.

\subsection{Experimental validation}

To confirm the results of the network analysis and verify the key targets of BYHWD brain protection, we selected three key targets (IL6, VEGFA and HIF1A) for pharmacological validation (Fig. 7). Western blot results showed that compared with the control group (no treatment), BYHWD could significantly inhibit the expression of IL6 and increased the expression of HIF1A and VEGFA $(p<0.05)$.

\section{Discussion}

Ischemic stroke is one of the leading causes of death and disability worldwide. Studies have shown that the injury mechanism of ischemic stroke includes apoptosis, necrosis, inflammation, immune regulation, oxidative stress, etc[17]. However, no effective treatment has been found to prevent damage to the brain 
in such cases except tissue plasminogen activator. This single compound or single target drug has limited effect on ischemic stroke. Therefore, a promising treatment approach for ischemic stroke is utilization of multiple-component agents with multiple targets[18].

Buyang huanwu decoction has the functions of supplementing qi, activating blood circulation and clearing collaterals. As a classic prescription for treating qi deficiency and blood stasis, it has been attached great importance by doctors of all generations. Traditional Chinese medicine cures disease through multiapproach, multi-target and integerconcept. It has unique advantages for thetreatment of complex diseases, but because of the complex composition of traditional Chinese medicine compound to make its modernization process slow. Furthermore, network pharmacology has provided a new way for exploring pharmacological mechanisms of TCMs. many scholars have been made an effort to apply network pharmacology to explore the complex of ingredients, targets, and mechanisms of herbal formulas[19].

In this study, network pharmacology was used to investigate the pharmacological mechanism of BYHWD related to cerebral ischemia, which improved the accuracy of targeted prediction to some extent. Network pharmacological analysis of BYHWD identified seven herbs, 42 compounds, and 79 target generegulated major pathways related to cerebral ischemia. In particular, the 16 compounds (baicalein, beta-carotene, Baicalin, kaempferol, luteolin, quercetin, hydroxysafflor yellow A, isorhamnetin, Bifendate, formononetin,Calycosin, AstragalosidelV, Stigmasterol, sitosterol, Z-ligustilide, Dihydrocapsaicin) were linked to more than five genes. A number of studies have reported that baicalein has potent neuroprote ctive properties under in vitro as well as in vivo systems[20]. Beta-carotene serve as an antioxidant, inhibiting free radical production. it may regulate cell growth anddeath[21]. Baicalin inhibited microglial cell activation and reduced inflammation, oxidative damage, and brain edema[22]. Additionally, Kaempferol has strong anti-inflammatory and antioxidant effects. Numerous scientific reports showed that it has beneficial role on different inflammatory-related diseases such as cardiovascular, and neurode generative diseases. Luteolin suppresses inflammation in the brain tissues and regulates different cell signaling pathways[23]. Quercetin has antioxidant stress and neuroprotective effects[24]. moreover, Hydroxysafflor yellow A protects brain microvascular endothelial cells (BMECs) against oxygen glucose deprivation/reoxygenation(OGD/R) induced injury by inhibiting autophagy via the Class IPI3K/Akt/mTOR signaling pathway[25]. Treatment of experimental stroke mice with isorhamnetin attenuated cerebral edema, improved blood-brain barrier function, and upregulated gene expression of tight junction proteins including occludin, ZO-1, and claudin-5[26]. Furthermore, calycosin protected the brain against ischemic injury through inhibiting calpain activation[27]. Dihydrocapsaicin treated cerebral I/R rats attenuate cerebral and BBB damage through inhibition of oxidative stress and inflammatory pathways[28]. In a word, these findings suggest that the main components of BYHWD are effective for treating cerebral ischemia.

Genes with high degrees of differential articulation were actually acquired depending on PPI system analysis, IL6, TNF, VEGFA, HIF1A, MAPK1, MAPK3, JUN, STAT3, IL1B and IL10 were recognized as center genes. Interleukin-6 (IL-6) is a multi-functional cytokine with a wide range of biological activities such as regulation of the immune system, generation of acute phase reactions[29]. Vascular endothelial growth factor (VEGF) is a pleiotropic growth factor that is crucially involved in neurovascular remodeling in the ischemic brain. VEGF promotes angiogenesis, protects ischemic neurons from injury, has potent antiinflammatory actions, and promotes brain plasticity[30]. HIF1A regulates the expression of genes encoding molecules that participate in erythropoiesis, cell proliferation, and energy metabolism, and is closely associated with the regulation of neuronal survival in ischemia[31, 32]. HIF1A can up-regulate the gene expression of proteins related to the vascular system and promote the angiogenesis of VEGF and its receptors to increase blood flow and reduce ischemic injury[33]. TNF is a typical cytokine involved in the acute phase of systemic inflammation and is closely related to the severity of cerebral ischemia[34]. IL-10 is a potent anti-inflammatory mediator, and, if overexpressed, can suppress neuronal degeneration[35].

GO and KEGG pathway analysis to better comprehend the interaction and action pathway of target genes. In results, GO analyses revealed that target genes were majorly associated with the biological processes of positive regulation of transcription from RNA polymerase II promoter, inflammatory response, transcription, DNA-templated, negative regulation of apoptotic process, positive regulation of transcription, DNA-templated, angiogenesis, response to hypoxia, response to hypoxia. The enriched molecular function ontologies were dominated through DNA binding transcription factor activity, sequence-specific DNA binding,sequence-specific DNA binding, identical protein binding, heparin binding and heme binding so on. KEGG pathway analysis were primarily pertaining to TNF, IL-17, Apoptosis, PI3K-Akt, Toll-like receptor, MAPK, NF-kappa B and HIF-1 signaling pathway. Our results suggest that these pathways might interact to exert their combined effects against cerebral ischemia, which could explain the apparent effects of BYHWD.

Cerebral ischemia results in decreased cerebral blood flow, decreased oxygen supply, induced activation of the HIF-1 signaling pathway, and upregulated HIF1A expression, which contributes to the recovery of blood circulation in the penumbra after cerebral ischemia and the transport of glucose, and mediates hypoxia adaptation after hypoxia, and plays a protective role in promoting cell survival and inhibiting apoptosis in brain tissues[36]. MAPK and PI3K/AKT signaling pathways are the main signaling pathways related to apoptosis after cerebral ischemia. Currently, it is believed that MAPK signaling plays a dual role in the process of cell apoptosis, while PI3K/Akt signaling is an important cell survival signaling pathway. Multiple neurotrophic factors inhibit apoptosis by activating the PI3K/Akt signaling pathway, thus playing a protective role in the brain[37]. The PI3K/Akt signaling pathway is involved in the regulation of various intracellular signaling pathways and plays a key role in promoting cell survival and proliferation, anti-apoptosis, regulating glucose metabolism and protein synthesis[38]. Toll-like receptors (TLRS), as inflammatory signal receptors, play an important role in the inflammatory cascade reaction triggered by cerebral ischemia and are closely related to the expression of various inflammatory mediators. Therefore, it is of great significance to intervene the TLRs signaling pathway in the initial stage of inflammatory response to effectively reduce the inflammatory injury in the acute stage of ischemic stroke[39].

In addition, we verified three of the key targets (IL6, VEGFA and HIF1A) that predicted in the network by using western blot analysis. Therefore, the pharmacological mechanism of BYHWD in the treatment of cerebral ischemia can be more clearly verified. The results showed that compared with the control group (no treatment), BYHWD could significantly inhibit the expression of IL6 and increased the expression of HIF1A and VEGFA.

\section{Conclusion}


In summary, network pharmacology analysis of BYHWD identified 7 herbs, 42 compounds and 79 target genes associated to cerebral ischemia. The 10 key compounds were baicalein, beta-carotene, Baicalin, kaempferol, luteolin, quercetin, hydroxysafflor yellow A, isorhamnetin, Bifendate, formononetin,Calycosin, AstragalosidelV, Stigmasterol, sitosterol, Z-ligustilide, Dihydrocapsaicin. Based on the results of pathwayenrichment, we determined that effects of BYHWD against cerebral ischemia may be due to that ingredients of BYHWD can simultaneously target multiple pathways like TNF, IL-17, Apoptosis, PI3K-Akt, Toll-like receptor, MAPK, NF-kappa B and HIF-1 signaling pathway and so on. Also, genes with high degrees of differential articulation were actually acquired depending on PPI system analysis, IL6, TNF, VEGFA, HIF1A, MAPK1, MAPK3, JUN, STAT3, IL1B and IL10 were recognized as center genes. Our results indicate that compound-target gene networks can reveal close interactions between multi-components and multi-targets, and enhance our understanding of the potential effects of BYHWD in cerebral ischemia.

\section{Abbreviations}

BYHWD, Buyang Huanwu Decoction; ADME, Absorption, Distribution, Metabolism, and Excretion; AHA, American heart association; TCM, Traditional Chinese medicine; OB, oral bioavailability; DL, drug-likeness; KEGG, Kyoto Encyclopedia of Genes and Genomes; GO, Gene Ontology; PPI, protein-protein interaction; BMECs, Brain Microvascular Endothelial cells; OGD, Oxygen Glucose Deprivation;BP, biological process; MF, molecular function; CC, cellular component; IL-6, Interleukin-6; VEGFA, Vascular endothelial growth factor-A; HIF1A, Hypoxia-inducible factor 1-alpha; TLRS, Toll-like receptors.

\section{Declarations}

\section{Acknowledgements}

Not applicable.

\section{Author contributions:}

WK, LL, CJ, QY designed the experiments; WK, LL conducted experiments and researched literature; WK, CJ, QY, LR collected and analyzed the data; WK wrote the manuscript; WK, CJ, YZ revised the manuscript. DJ, FZ, DY, MY conducted data interpretation; YZ, ZE conducted Funds Collection. All authors commented on the results and approved the final manuscript.

\section{Funding}

This research was funded by National Natural Science Foundation of China (No. 81503280, 81573549).

\section{Availability of data and materials}

The datasets used and/or analysed during the current study are available from the corresponding author on reasonable request.

\section{Ethics approval and consent to participate}

Not applicable.

\section{Consent for publication}

Not applicable.

\section{Competing interests}

The authors declare no conflict of interest.

\section{References}

1. Del Sette M, Chiti A, Dinia L. Intraarterial Treatment for Acute Ischemic Stroke. N Engl J Med. 2015;372(12):1176-7.

2. Wang P, Shao BZ, Deng Z, Chen S, Yue Z, Miao CY. Autophagy in ischemic stroke. Prog Neurobiol. 2018 Apr - May;163-164:98-117.

3. Writing Group M, Mozaffarian D, Benjamin EJ, Go AS, Arnett DK, Blaha MJ, et al. Heart Disease and Stroke Statistics-2016 Update: A Report From the American Heart Association. Circulation. 2016 Jan;26(4):e38-60. 133(.

4. Hung IL, Hung YC, Wang LY, Hsu SF, Chen HJ, Tseng YJ, et al. Chinese Herbal Products for Ischemic Stroke. Am J Chin Med. 2015;43(7):1365-79.

5. Li JH, Liu AJ, Li HQ, Wang Y, Shang HC, Zheng GQ. Buyang huanwu decoction for healthcare: evidence-based theoretical interpretations of treating different diseases with the same method and target of vascularity. Evid Based Complement Alternat Med. 2014;2014:506783.

6. T X, Z SLYS. P, S L, F S, et al. Systematically characterize the absorbed effective substances of Wutou Decoction and their metabolic pathways in rat plasma using UHPLC-Q-TOF-MS combined with a target network pharmacological analysis. J Pharm Biomed Anal. 2017;141:95-107.

7. Kim SK, Lee S, Lee MK, Lee S. A systems pharmacology approach to investigate the mechanism of Oryeong-san formula for the treatment of hypertension. J Ethnopharmacol. 2019 Nov;15:244:112129.

8. Wang W, Liu T, Yang L, Ma Y, Dou F, Shi L, et al. Study on the multi-targets mechanism of triphala on cardio-cerebral vascular diseases based on network pharmacology. Biomed Pharmacother. 2019 Aug;116:108994. 
9. Zhang J, Yang B, Wang L, Liang R. Effects and mechanisms of Danshen-Shanzha herb-pair for atherosclerosis treatment using network pharmacology and experimental pharmacology. Journal of Ethnopharmacology: An Interdisciplinary Journal Devoted to Bioscientific Research on Indigenous Drugs. 2019;229:104-14.

10. A F, D S, S F, M K, M S, A R, et al. STRING v9.1: protein-protein interaction networks, with increased coverage and integration. Nucleic acids research. 2013;41:D808-15.

11. Sun Y, Xu DP, Qin Z, Wang PY, Hu BH, Yu JG, et al. Protective cerebrovascular effects of hydroxysafflor yellow A (HSYA) on ischemic stroke. Eur J Pharmacol. 2018 Jan;5:818:604-9.

12. Yin F, Zhou H, Fang Y, Li C, He Y, Yu L, et al. Astragaloside IV alleviates ischemia reperfusion-induced apoptosis by inhibiting the activation of key factors in death receptor pathway and mitochondrial pathway. J Ethnopharmacol. 2020 Feb;10:248:112319.

13. Fritsche KL. Linoleic acid, vegetable oils \& inflammation. Mo Med. 2014 Jan-Feb;111(1):41-3.

14. Zdunska K, Dana A, Kolodziejczak A, Rotsztejn H. Antioxidant Properties of Ferulic Acid and Its Possible Application. Skin Pharmacol Physiol. 2018;31(6):332-6.

15. Li J, Yu J, Ma H, Yang N, Li L, Zheng DD, et al. Intranasal Pretreatment with Z-Ligustilide, the Main Volatile Component of Rhizoma Chuanxiong, Confers Prophylaxis against Cerebral Ischemia via Nrf2 and HSP70 Signaling Pathways. J Agric Food Chem. 2017 Mar 1;65(8):1533-42.

16. Wei Y, Liu J, Zhang H, Du X, Luo Q, Sun J, et al. Ligustrazine attenuates inflammation and the associated chemokines and receptors in ovalbumineinduced mouse asthma model. Environ Toxicol Pharmacol. 2016 Sep;46:55-61.

17. Pandya RS, Mao L, Zhou H, Zhou S, Zeng J, Popp AJ, et al. Central nervous system agents for ischemic stroke: neuroprotection mechanisms. Cent Nerv Syst Agents Med Chem. 2011 Jun 1;11(2):81-97.

18. Zhang WW, Xu F, Wang D, Ye J, Cai SQ. Buyang Huanwu Decoction ameliorates ischemic stroke by modulating multiple targets with multiple components: In vitro evidences. Chin J Nat Med. 2018 Mar;16(3):194-202.

19. Lee AY, Park W, Kang TW, Cha MH, Chun JM. Network pharmacology-based prediction of active compounds and molecular targets in Yijin-Tang acting on hyperlipidaemia and atherosclerosis. J Ethnopharmacol. 2018 Jul;15:221:151-9.

20. SJ KSPDMK. P, S K. Baicalein as a potent neuroprotective agent: A review. Biomedicine pharmacotherapy = Biomedecine pharmacotherapie. 2017;95:1021-32.

21. P P. Can beta-carotene regulate cell growth by a redox mechanism? An answer from cultured cells. Biochimica et biophysica acta. 2005;1740(2):215-21.

22. Shi X, Fu Y, Zhang S, Ding H, Chen J. Baicalin Attenuates Subarachnoid Hemorrhagic Brain Injury by Modulating Blood-Brain Barrier Disruption, Inflammation, and Oxidative Damage in Mice. Oxid Med Cell Longev. 2017;2017:1401790.

23. SF N, O G NB, E S-S MD, K S-W, et al. Luteolin as an anti-inflammatory and neuroprotective agent: A brief review. Brain research bulletin. 2015;119:1-11.

24. Barreca D, Bellocco E, D'Onofrio G, Nabavi SF, Daglia M, Rastrelli L, et al. Neuroprotective Effects of Quercetin: From Chemistry to Medicine. CNS Neurol Disord Drug Targets. 2016;15(8):964-75.

25. Yang G, Wang N, Seto SW, Chang D, Liang H. Hydroxysafflor yellow a protects brain microvascular endothelial cells against oxygen glucose deprivation/reoxygenation injury: Involvement of inhibiting autophagy via class I PI3K/Akt/mTOR signaling pathway. Brain Res Bull. 2018 Jun;140:24357.

26. JJ Z, JQ S, SY P. K W. Treatment with Isorhamnetin Protects the Brain Against Ischemic Injury in Mice. Neurochem Res. 2016;41(8):1939-48.

27. Guo C, Ma Y, Ma S, Mu F, Deng J, Duan J, et al. The Role of TRPC6 in the Neuroprotection of Calycosin Against Cerebral Ischemic Injury. Sci Rep. 2017 Jun 8;7(1):3039.

28. Janyou A, Wicha P, Jittiwat J, Suksamrarn A, Tocharus C, Tocharus J. Dihydrocapsaicin Attenuates Blood Brain Barrier and Cerebral Damage in Focal Cerebral Ischemia/Reperfusion via Oxidative Stress and Inflammatory. Sci Rep. 2017 Sep;5(1):10556. 7 (.

29. Robson-Ansley P, Cockburn E, Walshe I, Stevenson E, Nimmo M. The effect of exercise on plasma soluble IL-6 receptor concentration: a dichotomous response. Exerc Immunol Rev. 2010;16:56-76.

30. Ma Y, Zechariah A, Qu Y, Hermann DM. Effects of vascular endothelial growth factor in ischemic stroke. J Neurosci Res. 2012 Oct;90(10):1873-82.

31. Ciurea AV, Palade C, Voinescu D, Nica DA. Subarachnoid hemorrhage and cerebral vasospasm - literature review. J Med Life. 2013 Jun;15(2):120-5. 6(.

32. Guo Y. Role of HIF-1a in regulating autophagic cell survival during cerebral ischemia reperfusion in rats. Oncotarget. 2017 Nov 17;8(58):98482-94.

33. Zhen-Zhen C, Xin G, Qi G, Hui Z, Lei W. Bu Yang Huan Wu Decoction prevents reperfusion injury following ischemic stroke in rats via inhibition of HIF-1 a, VEGF and promotion $\beta$-ENaC expression. Journal of ethnopharmacology. 2018.

34. Oliveira DMG, Aguiar LT, de Oliveira Limones MV, Gomes AG, da Silva LC, de Morais Faria CDC, et al. Aerobic Training Efficacy in Inflammation, Neurotrophins, and Function in Chronic Stroke Persons: A Randomized Controlled Trial Protocol. J Stroke Cerebrovasc Dis. 2019 Feb;28(2):418-24.

35. C MN, K N, Y N, A N-T SSS, et al. Mesenchymal Stem Cells Overexpressing Interleukin-10 Promote Neuroprotection in Experimental Acute Ischemic Stroke. Molecular therapy Methods clinical development. 2017;6:102-11.

36. Singh N, Sharma G, Mishra V. Hypoxia inducible factor-1: its potential role in cerebral ischemia. Cell Mol Neurobiol. 2012 May;32(4):491-507.

37. Franke TF. PI3K/Akt: getting it right matters. Oncogene. 2008 Oct 27;27(50):6473-88.

38. Gao X, Zhang H, Takahashi T, Hsieh J, Liao J, Steinberg GK, et al. The Akt signaling pathway contributes to postconditioning's protection against stroke; the protection is associated with the MAPK and PKC pathways. J Neurochem. 2008;105(3):943-55.

39. T E, HK E. Ischemia and reperfusion-from mechanism to translation. Nature medicine. 2011;17(11):1391-401. 


\section{Tables}

Due to technical limitations, Table 1 is provided in the Supplementary Files.

Table 2

Biological processes of potential target genes based on GO enrichment analysis.

\begin{tabular}{|c|c|c|c|}
\hline ID & Biological Process & Genes & P-Value \\
\hline GO:0045944 & $\begin{array}{l}\text { positive regulation of transcription from } \\
\text { RNA polymerase II promoter }\end{array}$ & $\begin{array}{l}\text { HMGB1,TNF,RELA,PPARG,TP53,IGF1, NFKBIA,RB1,CD40, SIRT1, IL10,VDR, } \\
\text { IL17A, APP,HIF1A,SP1,POU5F1,RIPK1 , JUN, MAPK3 }\end{array}$ & $6.60 \mathrm{E}-12$ \\
\hline GO:0006954 & inflammatory response & $\begin{array}{l}\text { PIK3CG, HMGB1, CCL2, PTGS2,RELA, TLR4, CD40, AGER, IL10, CXCL10, } \\
\text { IL17A, JAK2, NFE2L2, CD14 }\end{array}$ & 7.35E-11 \\
\hline GO:0000122 & $\begin{array}{l}\text { negative regulation of transcription from } \\
\text { RNA polymerase II promoter }\end{array}$ & $\begin{array}{l}\text { EGR1,VDR,TNF,POU5F1,RELA,JUN, PPARG, TP53, NFKB1, RB1, SIRT1, DDIT3, } \\
\text { EPO }\end{array}$ & 3.56E-07 \\
\hline GO:0006351 & transcription, DNA-templated & EGR1,VDR,HIF1A,CEBPB,JUN,MAPK3, PPARG, TP53, STAT3 & 5.98E-05 \\
\hline GO:0043066 & negative regulation of apoptotic process & CASP3, ALB, BCL2, TP53, IGF1, CAT, SIRT1, STAT3 & $1.78 \mathrm{E}-04$ \\
\hline GO:0045893 & $\begin{array}{l}\text { positive regulation of transcription, DNA- } \\
\text { templated }\end{array}$ & CEBPB,JUN, PPARG,NFKB1, NFE2L2, STAT3, EPO & $1.78 \mathrm{E}-04$ \\
\hline GO:0031663 & $\begin{array}{l}\text { lipopolysaccharide-mediated signaling } \\
\text { pathway }\end{array}$ & MAPK1, CCL2, TNF, MAPK3, NFKBIA, NOS3, TLR4 & $7.02 \mathrm{E}-10$ \\
\hline GO:0001525 & angiogenesis & PIK3CG,HIF1A,JUN,VEGFA,SERPINE, NOS3, SIRT1 & $2.14 \mathrm{E}-05$ \\
\hline GO:0042127 & regulation of cell proliferation & JUN,NFKBIA,JAK2,NOS2,CD40,PLAU, CXCL10 & $8.12 \mathrm{E}-05$ \\
\hline GO:0001666 & response to hypoxia & PLAT,VEGFA,NOS2,AGER, PLAU,EPO & $1.96 \mathrm{E}-05$ \\
\hline GO:0051092 & $\begin{array}{l}\text { positive regulation of NF-kappaB } \\
\text { transcription factor activity }\end{array}$ & TNF, RELA, RIPK1, CAT, CD40, AGER & $7.26 \mathrm{E}-05$ \\
\hline GO:0006915 & apoptotic process & APP, RIPK1, CYCS, TP53, RB1, EPO & $2.98 \mathrm{E}-04$ \\
\hline GO:0055114 & oxidation-reduction process & GPX1, NOS3, CAT, NOS2, SOD1, SOD2 & 0.001431211 \\
\hline GO:0006955 & immune response & CSF2, IL6, JUN, CD40, IL10, CXCL10 & 0.006954104 \\
\hline GO:0043491 & protein kinase B signaling & CSF2, CCL2, TNF, IGF1, CD40 & $1.21 \mathrm{E}-05$ \\
\hline GO:0071356 & cellular response to lipopolysaccharide & JUN, SERPINE1, NOS2, IL10, CXCL10 & $1.82 \mathrm{E}-04$ \\
\hline GO:0006979 & response to oxidative stress & GPX1, APP, PTGS2, APOE, MPO & $2.91 \mathrm{E}-04$ \\
\hline GO:0045766 & positive regulation of angiogenesis & HIF1A, VEGFA, SERPINE1, NOS3, SIRT1 & $6.75 \mathrm{E}-04$ \\
\hline GO:0043524 & $\begin{array}{l}\text { negative regulation of neuron apoptotic } \\
\text { process }\end{array}$ & CCL2, APOE, JUN, BCL2, SOD1 & $7.56 \mathrm{E}-04$ \\
\hline
\end{tabular}

\section{Table 3}

Functions of potential target genes based on KEGG pathway analysis. 


\begin{tabular}{|c|c|c|}
\hline ID & Pathway & Number of pathway gene \\
\hline hsa05167 & $\begin{array}{l}\text { Kaposi sarcoma- } \\
\text { associated } \\
\text { herpesvirus } \\
\text { infection }\end{array}$ & PTGS2/CALM1/PIK3CG/RELA/RB1/MAPK1/MAPK3/NFKBIA/CASP3/CSF2/CYCS/JUN/NFKB1/STAT3/TP53/VEGFA/IL6/CA \\
\hline hsa04668 & $\begin{array}{l}\text { TNF signaling } \\
\text { pathway }\end{array}$ & PTGS2/RELA/MMP9/MAPK1/MAPK3/NFKBIA/TNF/CASP3/CEBPB/CSF2/IL1B/JUN/NFKB1/IL6/ICAM1/CCL2/FOS/SELE/F \\
\hline hsa05163 & $\begin{array}{l}\text { Human } \\
\text { cytomegalovirus } \\
\text { infection }\end{array}$ & PTGS2/CALM1/RELA/RB1/MAPK1/MAPK3/NFKBIA/TNF/CASP3/CYCS/IL1B/NFKB1/STAT3/TP53/VEGFA/IL6/CASP9/CCL \\
\hline hsa04933 & $\begin{array}{l}\text { AGE-RAGE } \\
\text { signaling pathway } \\
\text { in diabetic } \\
\text { complications }\end{array}$ & RELA/MAPK1/MAPK3/TNF/BCL2/CASP3/IL1B/JUN/NFKB1/STAT3/VEGFA/IL6/ICAM1/CCL2/SELE/AGER/EGR1/NOS3/AC \\
\hline hsa04657 & $\begin{array}{l}\text { IL-17 signaling } \\
\text { pathway }\end{array}$ & PTGS2/RELA/MMP9/MAPK1/MAPK3/NFKBIA/TNF/CASP3/CEBPB/CSF2/IL1B/JUN/NFKB1/IL6/CCL2/FOS/CXCL10/FOSB, \\
\hline hsa05161 & Hepatitis B & RELA/MMP9/RB1/MAPK1/MAPK3/NFKBIA/TNF/BCL2/CASP3/CYCS/JUN/NFKB1/STAT3/TP53/IL6/TLR4/CASP9/FOS/CRF \\
\hline hsa05152 & Tuberculosis & CALM1/NOS2/RELA/MAPK1/MAPK3/TNF/BCL2/CASP3/CEBPB/CYCS/IL1B/NFKB1/IL6/TLR4/CASP9/CD14/VDR/IL10/CR \\
\hline hsa05145 & Toxoplasmosis & NOS2/PIK3CG/RELA/MAPK1/MAPK3/NFKBIA/TNF/BCL2/CASP3/CYCS/NFKB1/STAT3/TLR4/CASP9/IL10/CD40/HSPA1A/. \\
\hline hsa04210 & Apoptosis & RELA/MAPK1/MAPK3/NFKBIA/TNF/BCL2/CASP3/CYCS/JUN/NFKB1/TP53/CASP9/PARP1/BBC3/ATM/FOS/RIPK1/DDIT3 \\
\hline hsa05142 & $\begin{array}{l}\text { Chagas disease } \\
\text { (American } \\
\text { trypanosomiasis) }\end{array}$ & NOS2/RELA/MAPK1/MAPK3/NFKBIA/TNF/IL1B/JUN/NFKB1/IL6/TLR4/CCL2/FOS/IL10/ACE/SERPINE1/CCL3 \\
\hline hsa05418 & $\begin{array}{l}\text { Fluid shear stress } \\
\text { and } \\
\text { atherosclerosis }\end{array}$ & CALM1/RELA/MMP9/TNF/BCL2/IL1B/JUN/NFKB1/TP53/VEGFA/NFE2L2/ICAM1/CCL2/FOS/SELE/NOS3/PLAT \\
\hline hsa05202 & $\begin{array}{l}\text { Transcriptional } \\
\text { misregulation in } \\
\text { cancer }\end{array}$ & PPARG/RELA/MMP9/CEBPB/CSF2/IGF1/MPO/NFKB1/PLAU/TP53/IL6/ATM/CD14/CD40/SP1/DDIT3/PLAT \\
\hline hsa05169 & $\begin{array}{l}\text { Epstein-Barr } \\
\text { virus infection }\end{array}$ & RELA/RB1/NFKBIA/TNF/BCL2/CASP3/CYCS/JUN/NFKB1/STAT3/TP53/IL6/CASP9/ICAM1/RIPK1/CD40/CXCL10 \\
\hline hsa05166 & $\begin{array}{l}\text { Human } \\
\text { leukemia } \\
\text { infection }\end{array}$ & RELA/RB1/MAPK1/MAPK3/NFKBIA/TNF/CSF2/JUN/NFKB1/TP53/IL6/ICAM1/ATM/FOS/CD40/EGR1/CREB1 \\
\hline hsa04151 & $\begin{array}{l}\text { PI3K-Akt } \\
\text { signaling pathway }\end{array}$ & PIK3CG/RELA/MAPK1/MAPK3/BCL2/IGF1/NFKB1/TP53/VEGFA/IL6/TLR4/CASP9/NOS3/CREB1/JAK2/EPO/CHRM1 \\
\hline hsa04620 & $\begin{array}{l}\text { Toll-like receptor } \\
\text { signaling pathway }\end{array}$ & RELA/MAPK1/MAPK3/NFKBIA/TNF/IL1B/JUN/NFKB1/IL6/TLR4/CD14/FOS/RIPK1/CD40/CXCL10/CCL3 \\
\hline hsa05162 & Measles & RELA/NFKBIA/BCL2/CASP3/CYCS/IL1B/JUN/NFKB1/STAT3/TP53/IL6/TLR4/CASP9/BBC3/FOS/HSPA1A \\
\hline hsa05164 & Influenza A & RELA/MAPK1/MAPK3/NFKBIA/TNF/CASP3/CYCS/IL1B/NFKB1/IL6/TLR4/CASP9/ICAM1/CCL2/CXCL10/JAK2 \\
\hline hsa05170 & $\begin{array}{l}\text { Human } \\
\text { immunodeficiency } \\
\text { virus } 1 \text { infection }\end{array}$ & CALM1/RELA/MAPK1/MAPK3/NFKBIA/TNF/BCL2/CASP3/CYCS/JUN/NFKB1/TLR4/CASP9/ATM/FOS/RIPK1 \\
\hline hsa04010 & $\begin{array}{l}\text { MAPK signaling } \\
\text { pathway }\end{array}$ & RELA/MAPK1/MAPK3/TNF/CASP3/IGF1/IL1B/JUN/NFKB1/TP53/VEGFA/CD14/FOS/JUND/DDIT3/HSPA1A \\
\hline hsa05133 & Pertussis & CALM1/NOS2/RELA/MAPK1/MAPK3/TNF/CASP3/IL1B/JUN/NFKB1/IL6/TLR4/CD14/FOS/IL10 \\
\hline hsa04064 & $\begin{array}{l}\text { NF-kappa B } \\
\text { signaling pathway }\end{array}$ & PTGS2/RELA/NFKBIA/TNF/BCL2/IL1B/NFKB1/PLAU/TLR4/PARP1/ICAM1/ATM/CD14/RIPK1/CD40 \\
\hline hsa04066 & $\begin{array}{l}\text { HIF-1 signaling } \\
\text { pathway }\end{array}$ & NOS2/RELA/MAPK1/MAPK3/BCL2/IGF1/NFKB1/STAT3/VEGFA/IL6/TLR4/HIF1A/NOS3/SERPINE1/EPO \\
\hline
\end{tabular}

\section{Figures}




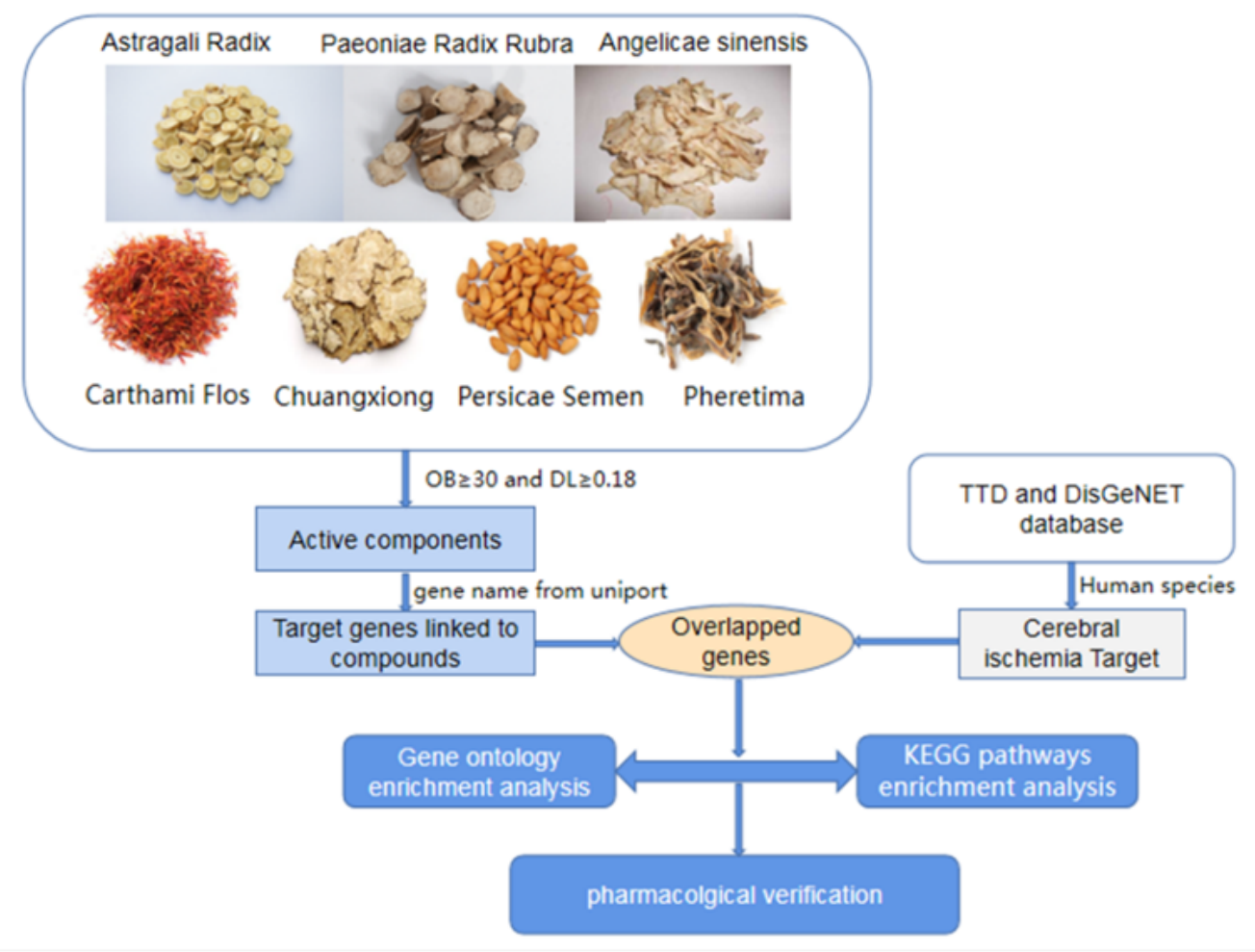

Figure 1

Workflow of network pharmacology analysis.

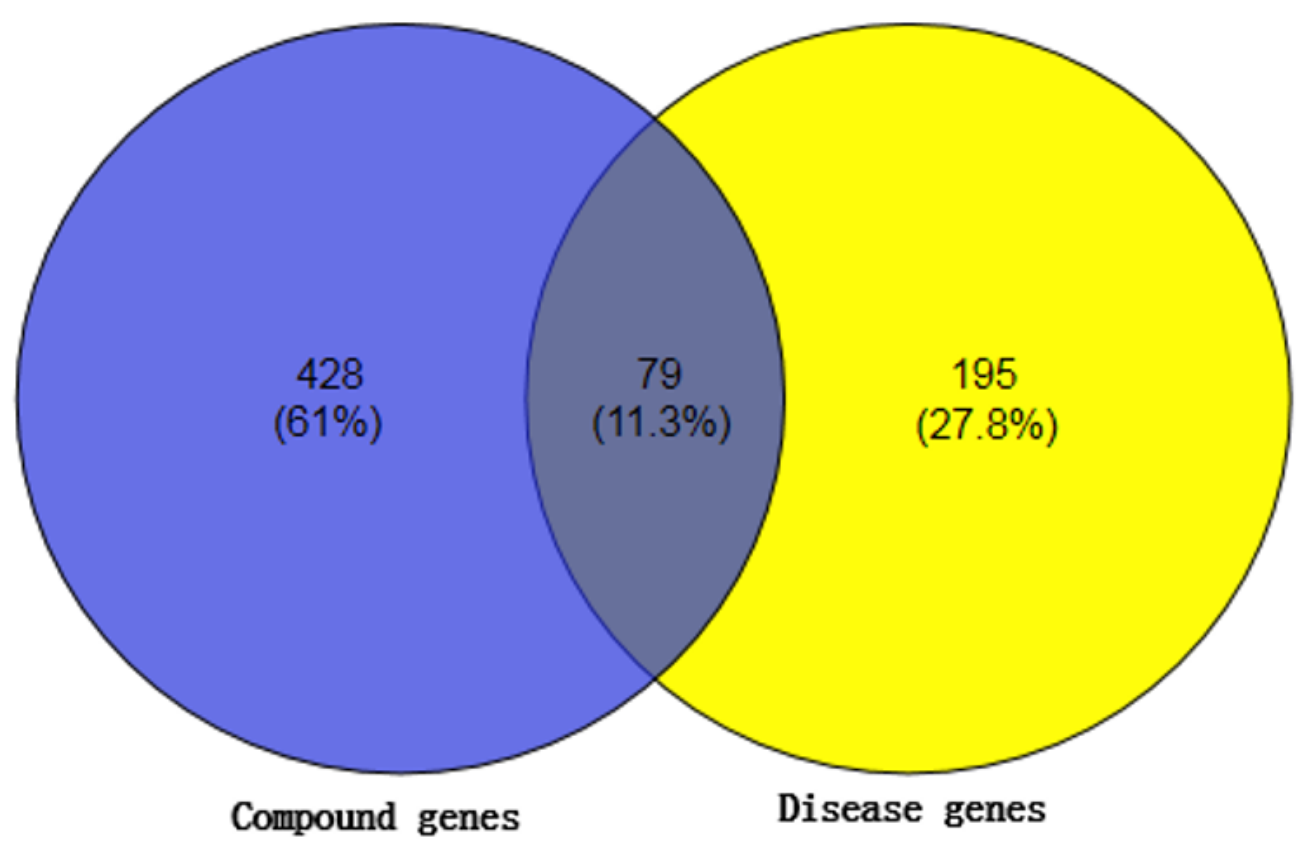

\section{Figure 3}

Venn diagram for compound genes and disease genes. 


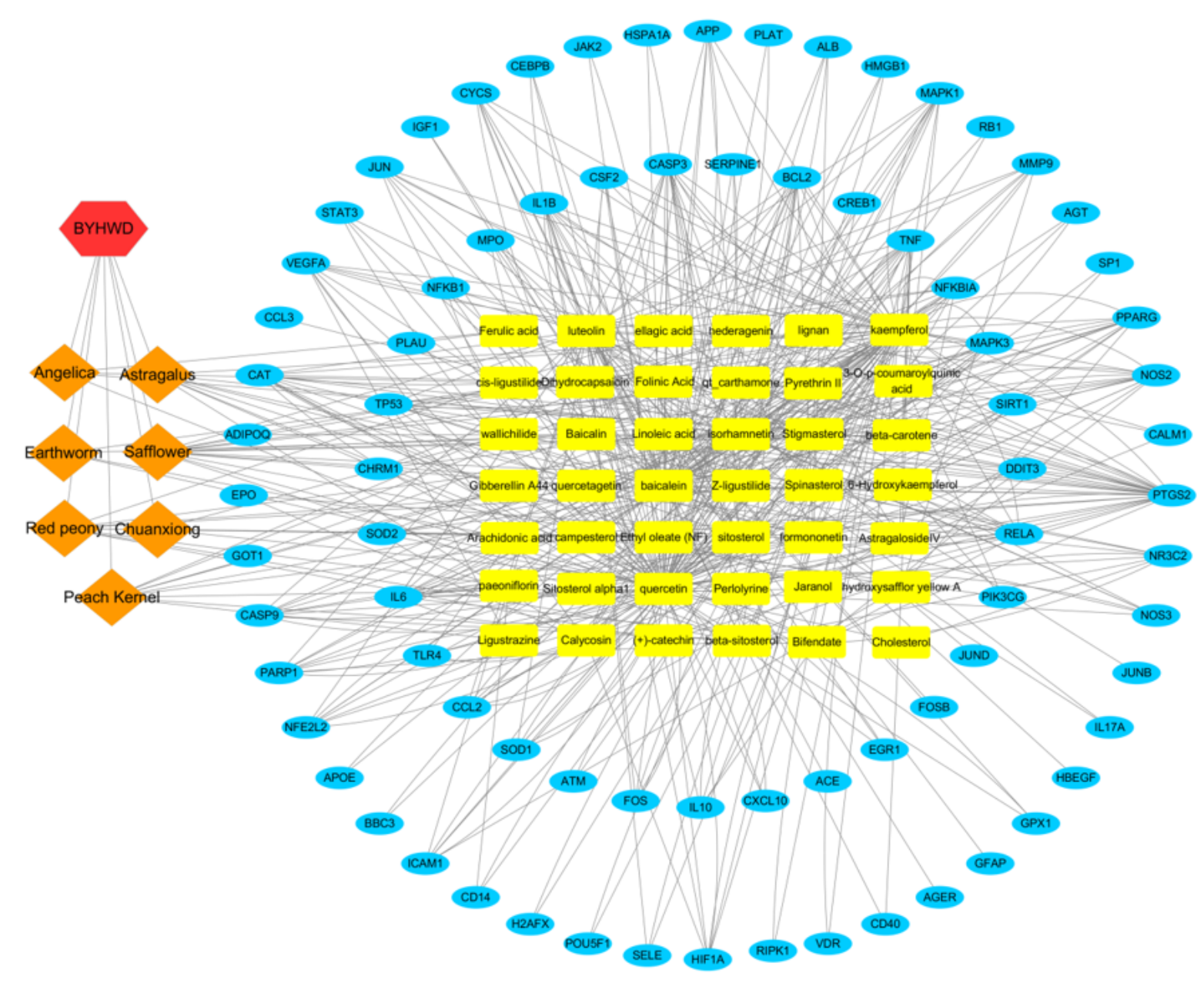

Figure 5

Network of BYHWD, seven herbal medicines and 42 compounds predicted to have 79 common potential protein targets. The blue ellipses represent the protein targets, orange diamonds delineate the herbal medicines, yellow ellipses represent the compounds and the red hexagon delineates the BYHWD. 


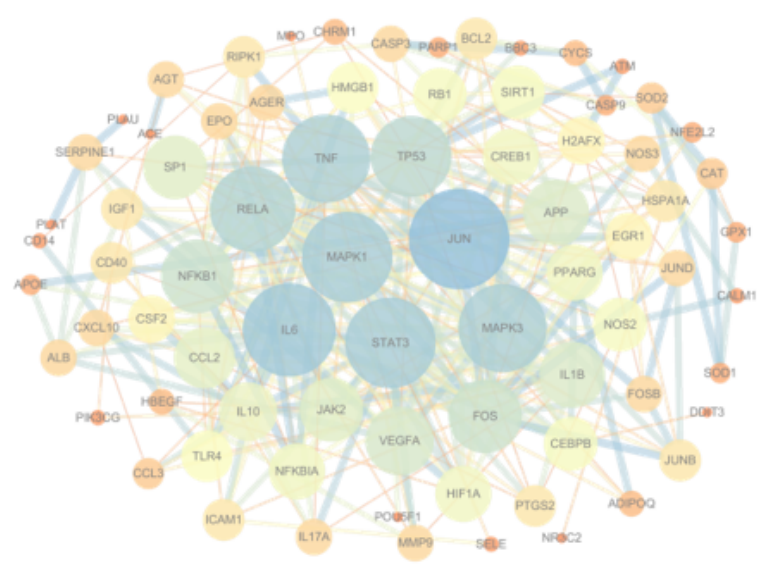

B

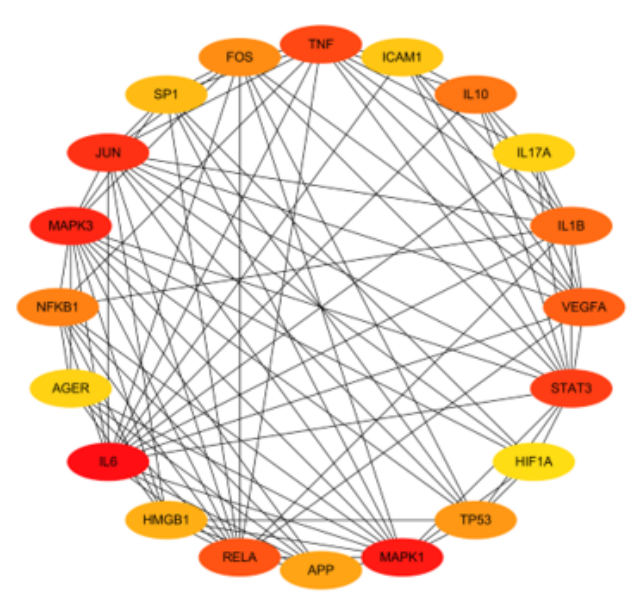

Figure 7

Protein-protein interaction (PPI) networks of active ingredients of BYHWD for the treatment of cerebral ischemia. (A) Each node represents the relevant gene, the edge means line thickness indicates the strength of data support. (B) Hub top 20 genes in PPI network, the darker the color, the higher the score.

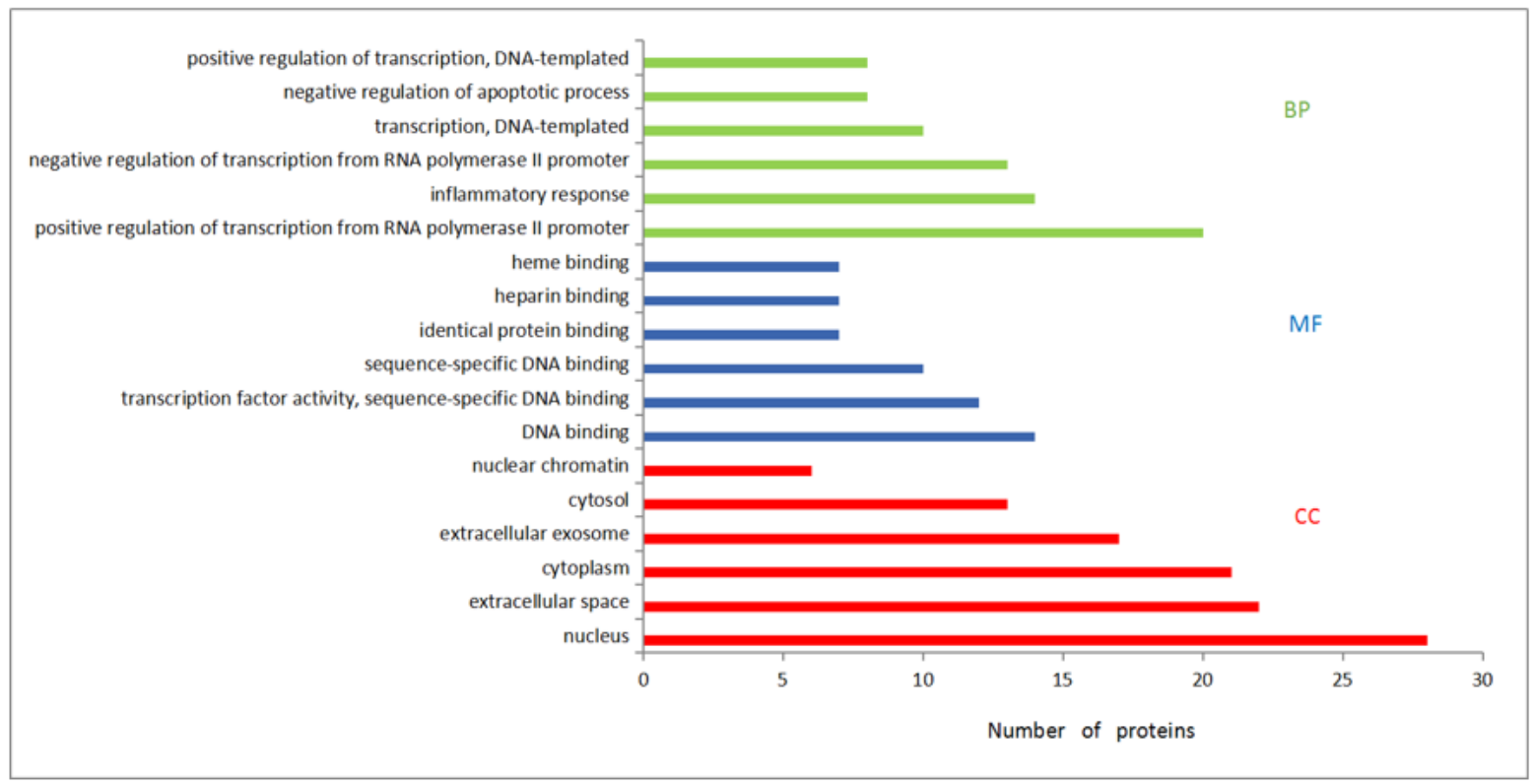

Figure 9 
GO analysis of candidate targets. Database showed the six remarkably enriched items in the biological processes (BP), cell component (CC) and molecular function (MF).

A

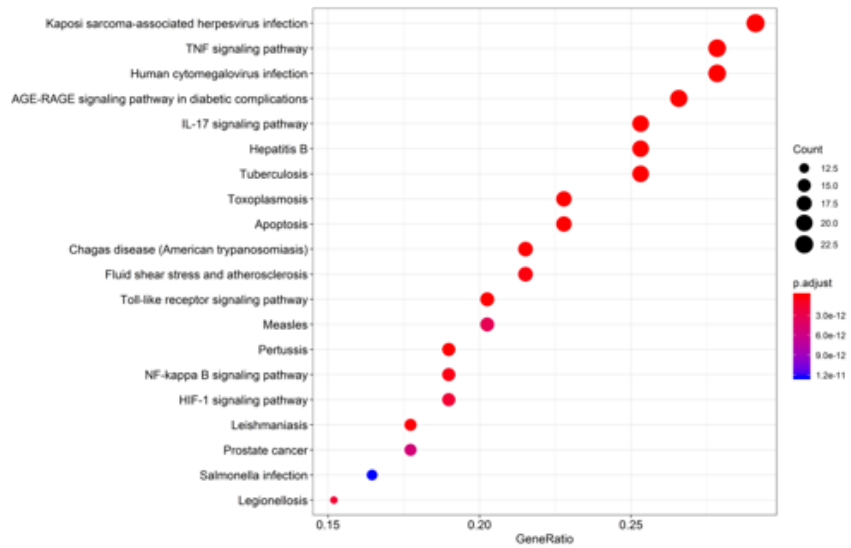

B

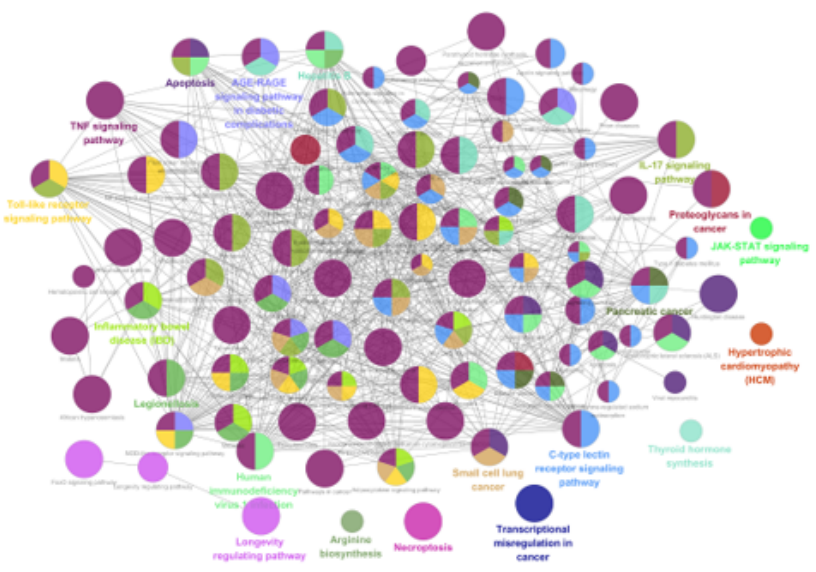

Figure 11

(A) KEGG pathways of target genes. (B) Main functional annotation clusters by Biocarta analysis. 

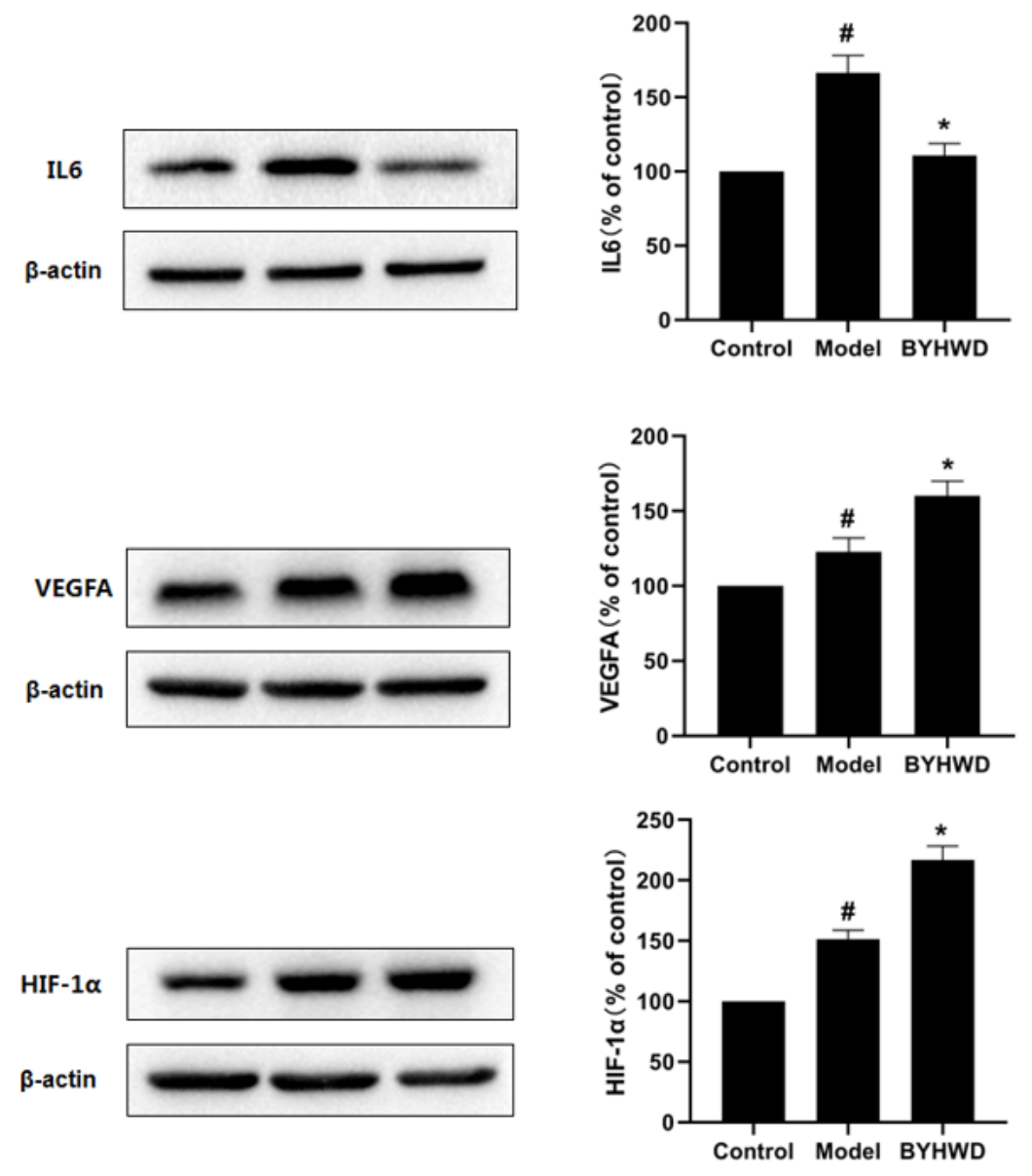

\section{Figure 13}

Effects of BYHWD on the levels of IL6, VEGFA and HIF-1a in BMECs. ( \#p<0.05compared with control group; * $<<0.05$ compared with model group).

\section{Supplementary Files}

This is a list of supplementary files associated with this preprint. Click to download.

- Table.docx

- Table.docx 